Competition between the modulation instability and stimulated Brillouin scattering in a broadband slow light device

This article has been downloaded from IOPscience. Please scroll down to see the full text article.

2010 J. Opt. 12104019

(http://iopscience.iop.org/2040-8986/12/10/104019)

View the table of contents for this issue, or go to the journal homepage for more

Download details:

IP Address: 152.3.237.20

The article was downloaded on 19/01/2012 at 18:16

Please note that terms and conditions apply. 


\title{
Competition between the modulation instability and stimulated Brillouin scattering in a broadband slow light device
}

\author{
Yunhui Zhu ${ }^{1}$, E Cabrera-Granado ${ }^{1}$, Oscar G Calderon ${ }^{2}$, \\ Sonia Melle ${ }^{2}$, Yoshitomo Okawachi ${ }^{3}$, Alexander L Gaeta ${ }^{3}$ and \\ Daniel J Gauthier ${ }^{1}$ \\ ${ }^{1}$ Department of Physics and the Fitzpatrick Institute for Photonics, Duke University, Durham, \\ NC 27708, USA \\ ${ }^{2}$ Escuela Universitaria de Optica, Universidad Complutense de Madrid, C/Arcos de Jalón s/n, \\ 28037 Madrid, Spain \\ ${ }^{3}$ School of Applied and Engineering Physics, Cornell University, Ithaca, NY 14853, USA \\ E-mail: yz65@phy.duke.edu
}

Received 20 April 2010, accepted for publication 2 July 2010

Published 24 September 2010

Online at stacks.iop.org/JOpt/12/104019

\begin{abstract}
We observe competition between the modulation instability (MI) and stimulated Brillouin scattering (SBS) in a $9.2 \mathrm{GHz}$ broadband SBS slow light device, in which a standard $20 \mathrm{~km}$ long single-mode LEAF fibre is used as the SBS medium. We find that MI is dominant and depletes most of the pump power when we use an intense pump beam at $\sim 1.55 \mu \mathrm{m}$, where the LEAF fibre is anomalously dispersive. The dominance of the MI in the LEAF-fibre-based system suppresses the SBS gain, degrading the SBS slow light delay and limiting the SBS gain-bandwidth to $125 \mathrm{~dB} \mathrm{GHz}$. In a dispersion-shifted highly nonlinear fibre, the SBS slow light delay is improved due to the suppression of the MI, resulting in a gain-bandwidth product of $344 \mathrm{~dB} \mathrm{GHz}$, limited by our available pump power of $0.82 \mathrm{~W}$.
\end{abstract}

Keywords: slow light, nonlinear fibre optics, stimulated Brillouin scattering, modulation instability

(Some figures in this article are in colour only in the electronic version)

\section{Introduction}

Slow light refers to the dramatic reduction of the group velocity $v_{\mathrm{g}}$ for optical pulses propagating through a dispersive material [1]. Among various slow light approaches, stimulated-Brillouin-scattering-based slow light in singlemode optical fibres has attracted much interest. Because of the optically controllable delay time and tunability of the bandwidth [2, 3], stimulated Brillouin scattering (SBS) slow light devices have great potential for all-optical applications such as network buffering, optical packet switching, and data synchronization $[4,5]$. Fractional delays as large as 3 have been demonstrated recently [6].

In SBS slow light systems, the bandwidth of the device is determined by the linewidth of the resonance. The intrinsic linewidth $\Gamma_{\mathrm{B}}$ of the resonance in normal single-mode fibre is $40 \mathrm{MHz}$ (FWHM), which is determined by the decay rate of acoustic phonons in the optical fibre. The data rate in such a system is limited to $\sim \Gamma_{\mathrm{B}}$, which is insufficient for modern optical communication applications. To solve this problem, broadband SBS slow light has been developed, in which the linewidth of the SBS resonance is broadened using a multifrequency laser source as the pump beam [7, 8].

Herráez et al [9] first demonstrated tailoring of the pump spectrum using direct modulation of the injecting current of the pump laser. Since then, a number of research groups have successfully broadened the bandwidth of the SBS slow light up to tens of $\mathrm{GHz}$, which is much larger than the intrinsic linewidth of the SBS resonance $[6,10,11]$. However, as we broaden the pump-beam spectrum, its power spectral density 


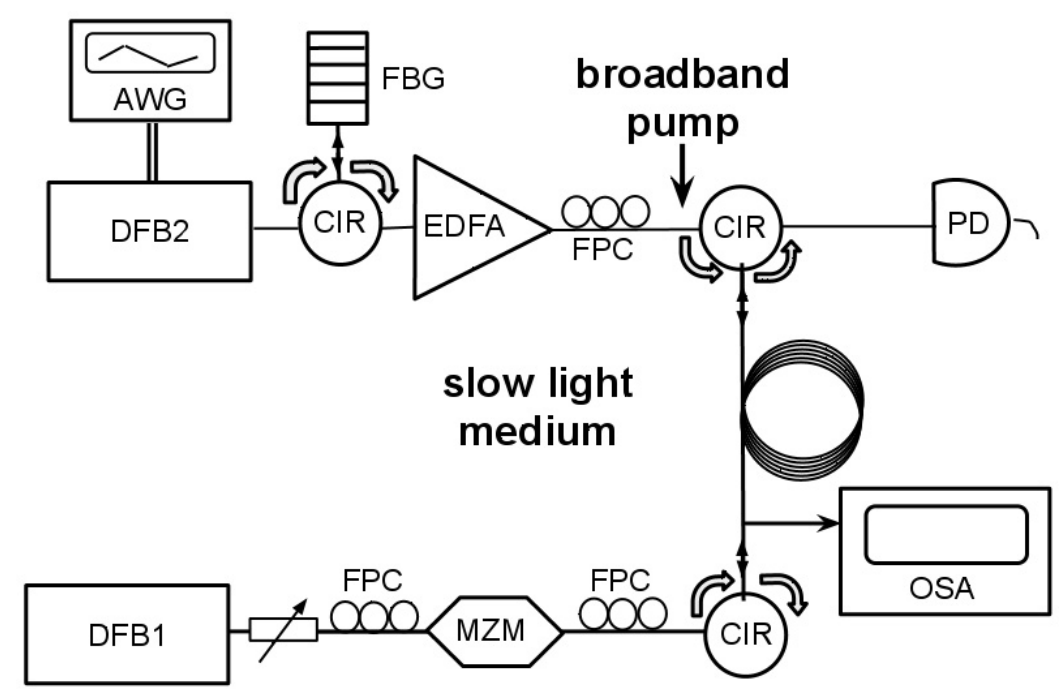

Figure 1. Experimental setup. The injection current of the pump laser (DFB2) is modulated by an arbitrary wavefunction generator (AWG). The current modulation function is tailored to produce a flat-topped broadened gain profile [6]. A fibre bragg grating (FBG) (bandwidth $\Delta v=24 \mathrm{GHz}$, central wavelength $\sim 1.55 \mu \mathrm{m}$ ) is used to filter out unwanted frequency sidebands from the DFB laser. An EDFA amplifies the pump beam before it is injected into the fibre via a circulator. The signal beam (DFB1) is modulated with a Mach-Zehnder modulator (MZM) and is injected from the other end of the fibre via a circulator. Fibre polarization controllers (FPC) are used to match the polarization orientations of the pump and signal beams. A photodiode (PD) measures the output power of the signal beam and an optical spectrum analyser (OSA) measures the spectrum of the output pump beam from the end of the fibre.

decreases, thereby suppressing the SBS gain. As a result, the slow light delay, which is proportional to the SBS gain, also decreases. Therefore, the power of the pump beam must be increased to compensate for the reduction of gain at larger bandwidths [12]. As the power increases, other nonlinear optical processes can become important if their response time is fast in comparison to the inverse of the pump-beam spectral width. For example, stimulated Raman scattering (SRS) and the modulation instability (MI) have response times of $\sim 1 \mathrm{ps}[13]$ and $\sim 10 \mathrm{fs}[14]$ respectively, and hence are not affected by pump broadening of several GHz. Eventually, as the bandwidth of the pump is increased and the power increased in proportion, SRS and/or the MI becomes more efficient, potentially robbing pump power and limiting the SBS gain.

Olsson et al [15] predict that SRS dominates over SBS for pump bandwidths larger than $20 \mathrm{GHz}$ for a gain of $20 \mathrm{~dB}$. No experiment validation of this prediction has been made to the best of our knowledge. On the contrary, our experiment shows that the MI is the dominant competing process in a $9.2 \mathrm{GHz}-$ bandwidth SBS slow light device, which limits the SBS gainbandwidth product.

In this paper, we study the competing effects and the related limitations on broadband SBS slow light. First, we introduce our broadband SBS slow light experiment and describe the competing processes that can possibly suppress the SBS process. We then verify that SBS is suppressed due to the MI in our broadband SBS device, where a standard singlemode LEAF fibre is used as the SBS medium. We focus here on the LEAF fibre because it is a readily available, relatively inexpensive fibre used in long-haul communication systems and hence is likely to be selected for SBS slow light devices. We also use a wavelength of $1.55 \mu \mathrm{m}$ because it is in the centre of the telecommunication band. As a solution to the problem, we show that the MI and the associated SBS slow light degradation are eliminated by using a dispersion-shifted fibre that has normal dispersion at the $1.55 \mu \mathrm{m}$ wavelength window. Finally, we compare the SBS gain-bandwidth product limit imposed by the MI with the limit imposed by SRS following the approach of Olsson et al [15] in our system.

\section{Broadband SBS slow light and the competing processes}

In a broadband SBS slow light device, an optical pulse (the signal) is delayed by interacting with a counterpropagating broadband pump beam via the SBS process. As shown in figure 1, two beams (signal and pump) counterpropagate through the slow light medium (fibre), where they interact and create low-frequency acoustic waves via electrostriction. These acoustic phonons, in turn, scatter the optical beams via Brillouin scattering. When the frequency of the signal beam is tuned to the Stokes line, that is, down-shifted by the Brillouin frequency $\Omega_{\mathrm{B}}$ from the frequency of the pump beam, the opto-acoustic coupling becomes strong, and light from the pump beam is efficiently scattered into the signal beam, inducing a gain resonance and giving rise to a variation in the refractive index in a narrow frequency range around the resonance frequency, which results in a small $v_{\mathrm{g}}$ for the signal beam [16].

Consider an optical pulse propagating through a fibre of length $L$, the transit time $T_{\mathrm{g}}$ is approximately given by $[17,18]$

$$
T_{\mathrm{g}}=\frac{L}{v_{\mathrm{g}}}=\frac{L n_{\mathrm{g}}}{c},
$$

where

$$
n_{\mathrm{g}}=n+v \frac{\mathrm{d} n}{\mathrm{~d} v}
$$


is the group index, $n$ is the refractive index, $v$ is the frequency (in cycles $\mathrm{s}^{-1}$ ) and $c$ is the speed of light in vacuum. The delay time $T_{\mathrm{d}}$ in a slow light system is defined as the difference in the transit time through the medium with and without the slow light effect, namely

$$
T_{\mathrm{d}}=T_{\mathrm{g}}-T_{\mathrm{g} 0}=\frac{L}{c}\left(n_{\mathrm{g}}-n_{\mathrm{g} 0}\right),
$$

where $n_{\mathrm{g}}$ and $n_{\mathrm{g} 0}$ are the group indices with and without slow light effect, respectively.

In our broadband SBS slow light experiment, a currentmodulated distributed feedback laser (DFB) is used to increase the linewidth of the SBS resonance. By designing the modulation function, we obtain a square-shaped pump-beam spectrum [6]. The broadened complex SBS field gain profile $\tilde{g}(v)$ results from the convolution of the intrinsic complex SBS gain spectrum $\tilde{g_{0}}(v)$ with the intensity spectrum $i_{i}\left(v_{p}\right)$ of the input pump beam, which is given by $[12,18]$

$$
\begin{aligned}
\tilde{g}(v) & =\tilde{g_{0}}(v) \otimes i_{i}\left(v_{p}\right) \\
& =\int_{-\infty}^{\infty} \frac{\left(g_{0} P_{\mathrm{i}} / A_{\mathrm{eff}} \Gamma\right) \operatorname{rect}\left[\left(v_{p}-v_{p 0}\right) / \Gamma\right]}{1-\mathrm{i}\left(v+\Omega_{\mathrm{B}}-v_{p}\right) /\left(\Gamma_{\mathrm{B}} / 2\right)} \mathrm{d} v_{p},
\end{aligned}
$$

where

$$
\operatorname{rect}(x)= \begin{cases}1 & |x|<1 / 2 \\ 0 & |x|>1 / 2\end{cases}
$$

$g_{0}$ is the line centre SBS amplitude gain factor (a constant determined by the material), $P_{\mathrm{i}}=\int i_{i}\left(v_{p}\right) \mathrm{d} v_{p} A_{\text {eff }}$ is the input power of the pump beam, $A_{\text {eff }}$ is the effective cross-section area of the fibre, $\Gamma=9.2 \mathrm{GHz}$ is the bandwidth (FWHM) of the pump spectrum and $v_{p 0}$ is the central frequency of the pump-beam spectrum. Equation (4) is obtained in the so-called undepleted pump approximation, where the pump-beam power $P_{\mathrm{i}}$ is constant along the fibre.

The gain profile of the broadened SBS resonance is obtained from the real part of the integral in (5) and is given by

$$
\begin{aligned}
g(\delta) & =\frac{\Gamma_{\mathrm{B}}}{2 \Gamma} \frac{g_{0} P_{\mathrm{i}}}{A_{\mathrm{eff}}}\left[\arctan \left(\frac{\delta+\Gamma / 2}{\Gamma_{\mathrm{B}} / 2}\right)-\arctan \left(\frac{\delta-\Gamma / 2}{\Gamma_{\mathrm{B}} / 2}\right)\right] \\
& \approx \frac{\pi \Gamma_{\mathrm{B}}}{2 \Gamma} \frac{g_{0} P_{\mathrm{i}}}{A_{\mathrm{eff}}} \operatorname{rect}(\delta / \Gamma) \quad \text { when } \Gamma \gg \Gamma_{\mathrm{B}}
\end{aligned}
$$

where $\delta=v+\Omega_{\mathrm{B}}-v_{p 0}$ is the signal beam detuning from resonance. We see that the bandwidth of the resonance is broadened to $\Gamma$. However, $g(\delta)$ scales inversely proportional to the bandwidth $\Gamma$ and more input power $P_{\mathrm{i}}$ is required to compensate for the loss in gain.

The refractive index $n$ associated with the SBS process is obtained from the imaginary part of (5) and is given by

$$
n(\delta)=n_{0}+\frac{c}{2 \pi v} \frac{\Gamma_{\mathrm{B}}}{4 \Gamma} \frac{g_{0} P_{\mathrm{i}}}{A_{\mathrm{eff}}} \ln \frac{1+\left[(\delta+\Gamma / 2) /\left(\Gamma_{\mathrm{B}} / 2\right)\right]^{2}}{1+\left[(\delta-\Gamma / 2) /\left(\Gamma_{\mathrm{B}} / 2\right)\right]^{2}},
$$

where $n_{0}$ is the refractive index without the SBS process. The group refractive index $n_{\mathrm{g}}$ at zero detuning $(\delta=0)$ is then determined using equations (2) and (9) to obtain

$n_{\mathrm{g}}=n+\left.v \frac{\mathrm{d} n}{\mathrm{~d} v}\right|_{\delta=0} \approx n_{\mathrm{g} 0}+\frac{c}{\pi} \frac{\Gamma_{\mathrm{B}}}{\Gamma^{2}} \frac{g_{0} P_{\mathrm{i}}}{A_{\mathrm{eff}}} \quad$ when $\Gamma \gg \Gamma_{\mathrm{B}}$.
Because we assume that the pump power $P_{\mathrm{i}}$ does not change over the fibre length $L$, the delay time of an on-resonance signal pulse whose spectral bandwidth is much smaller than that of the broadband SBS resonance is given approximately by

$$
T_{\mathrm{d}}=\frac{L}{c}\left(n_{\mathrm{g}}-n_{\mathrm{g} 0}\right) \approx \frac{\Gamma_{\mathrm{B}}}{\pi \Gamma^{2}} \frac{g_{0} P_{\mathrm{i}} L}{A_{\mathrm{eff}}} .
$$

Note that the power gain experienced by the probe beam is given by

$$
\begin{aligned}
G= & \ln \frac{P_{\mathrm{s}}}{P_{\mathrm{s} 0}}=2 g(0) L=2 \frac{\Gamma_{\mathrm{B}}}{\Gamma} \frac{g_{0} P_{\mathrm{i}} L}{A_{\mathrm{eff}}} \arctan \left(\Gamma / \Gamma_{\mathrm{B}}\right) \\
& \approx \frac{\pi \Gamma_{\mathrm{B}}}{\Gamma} \frac{g_{0} P_{\mathrm{i}} L}{A_{\mathrm{eff}}} \quad \text { when } \Gamma \gg \Gamma_{\mathrm{B}},
\end{aligned}
$$

where $P_{\mathrm{s}}$ and $P_{\mathrm{s} 0}$ are the output powers of the signal beam with and without the pump beam, respectively. If attenuation in the fibre is considered, the effective length $L_{\text {eff }}=[1-$ $\exp (-\alpha L)] / \alpha$ is used in place of length $L$ in equations (11)(13), where $\alpha$ is the attenuation coefficient. It follows from equations (11) and (13) that

$$
T_{\mathrm{d}}=\frac{G}{\pi^{2} \Gamma} \quad \text { when } \Gamma \gg \Gamma_{\mathrm{B}} .
$$

We see that the delay time $T_{\mathrm{d}}$ is proportional to the gain $G$, which is proportional to the pump power $P_{\mathrm{i}}$ and inversely proportional to the pump spectral bandwidth $\Gamma$. As a result, we are able to control the delay time $T_{\mathrm{d}}$ by adjusting the pump power.

There are a number of effects that limit the SBS gain $G$, thereby limiting the SBS slow light delay. One ultimate limitation on the SBS slow light delay is SBS gain saturation caused by pump depletion. It takes place when the Stokes beam becomes so strong that most of the power contained in the pump beam is transferred to it. Two different saturation processes are involved. One occurs when the initial probe beam is strong; the amplified probe beam grows quickly and depletes the pump beam even for moderate $G$. The other one takes place when $G$ approaches a threshold value $G_{\text {th }}$, found to be $\sim 10$ in single-mode fibres [19], where spontaneous Brillouin scattering from thermal fluctuations is amplified and becomes sufficiently large, depleting the pump power even in the absence of a probe beam. When the pump beam is depleted, the undepleted pump assumption is no longer valid. As a result, $G$, and thus the delay time $T_{\mathrm{d}}$, no longer grow with increasing pump power $[16,19]$.

Stimulated Raman scattering (SRS) is another competing nonlinear effect in which a pump beam is scattered by highfrequency optical phonons [14]. As a result, the frequency at which the SRS gain resonance occurs is down-shifted by $\sim 13 \mathrm{THz}$ from the frequency of the pump beam in single-mode fibres. In the presence of a strong pump beam, the Stokes beam initiated by spontaneous Raman scattering is amplified exponentially via the SRS process. When the SRS gain reaches a threshold value of $\sim 10$ in single-mode fibres [14], most of the pump power is transferred to the Stokes beam.

The SRS gain with a monochromatic pump is typically two orders of magnitude smaller than the SBS gain. However, as a result of the fast response time of SRS in single-mode 
Table 1. Parameters of the fibres used in the experiment.

\begin{tabular}{llllll}
\hline & $A_{\text {eff }}\left(\mu \mathrm{m}^{2}\right)$ & $L_{\text {eff }}(\mathrm{km})$ & $G / P_{\mathrm{i}}$ (linear region) $\left(\mathrm{W}^{-1}\right)$ & $\gamma L_{\text {eff }}\left(\mathrm{W}^{-1}\right)$ & $\beta_{2}\left(\mathrm{ps}^{2} \mathrm{~km}^{-1}\right)$ \\
\hline LEAF & 72 & 12.8 & 10.5 & 17.9 & -5.29 \\
HNLF & 11.7 & 1.64 & 11.1 & 14.3 & 0.08 \\
\hline
\end{tabular}

fibres ( $<1$ ps) [13], the Raman gain is not affected by the spectral broadening of the pump beam up to the value of $9.2 \mathrm{GHz}$ used in our experiment, whereas the SBS gain is inversely proportional to the bandwidth of the pump as shown in (13). As the pump bandwidth increases, the SBS gain will eventually match the SRS gain, setting the scale for competition between these processes.

MI is yet another process that can compete with the broadband SBS process. MI results from the interplay between the nonlinear Kerr effect and material dispersion. It is a fourwave-mixing process where two copropagating photons of the same frequency are converted into a frequency up-shifted and down-shifted photon pair [14, 20]. As a result, the MI broadens the spectrum of continuous-wave (cw) or quasi-cw beams, even turning a continuous-wave beam into a train of pulses [20-22]. In the presence of a strong cw or quasi-cw beam propagating through the fibre, noise components in the vicinity of peaks of the MI gain experience exponential amplification, which leads to the creation of two symmetric spectral side lobes $[14,20]$. The gain profile of the MI is given by [14]

$$
G_{\mathrm{MI}}(v)=4 \pi^{2}\left|\beta_{2} v\right| \sqrt{2 \Omega_{\text {peak }}^{2}-v^{2}} L,
$$

where $\Omega_{\text {peak }}$ (in cycles $\mathrm{s}^{-1}$ ) is the frequency shift at which the maximum gain is obtained, and $\beta_{2}$ is the group velocity dispersion (GVD) parameter. Here [14],

$$
\Omega_{\text {peak }}= \pm \frac{1}{2 \pi} \sqrt{\frac{2 \gamma P_{\mathrm{i}}}{\left|\beta_{2}\right|}},
$$

where $\gamma$ is the nonlinear parameter. Similar to SRS, the MI is not affected by the pump broadening of $9.2 \mathrm{GHz}$ due to its fast response time $(<10 \mathrm{fs})$. We will show that $\mathrm{MI}$ is the dominant competing effect in our SBS experiment at high input pump power $P_{\mathrm{i}}$ in a LEAF fibre.

\section{Experiment results}

In the experiment, as shown in figure 1, a distributed feedback (DFB) laser operating at $\sim 1.55 \mu \mathrm{m}$ is used as the pump source. We modulate the injecting current of the DFB laser with a modified triangle function so that the output beam of the DFB laser has a square-shaped spectrum with a bandwidth of 9.2 GHz [6]. The output of the DFB laser is then amplified with an erbium-doped fibre amplifier (EDFA) to provide enough pump power for the broadband SBS process. The EDFA also controls the input pump power and thereby controls the SBS gain. Another DFB laser is used to generate the signal beam, which is tuned to the SBS resonance. To avoid probe-induced SBS gain saturation, the signal beam is attenuated to a power of $2 \mu \mathrm{W}$ before it is injected into the SBS medium, where it counterpropagates and interacts with the pump beam via the SBS process. The amplified and delayed signal is detected at the output. The gain $G$ is obtained by measuring the output powers of the signal beam with the pump beam on and off.

We use two different fibres as the SBS medium in the experiment, a $20 \mathrm{~km}$ long LEAF fibre from Corning and a $2 \mathrm{~km}$ long highly nonlinear fibre (HNLF) fibre from OFS. The parameters of the fibres are shown in table 1 . The GVD parameter $\beta_{2}$ is measured by the time-of-flight method [24], in which the group velocity as function of the wavelength is measured by recording the transit time for optical pulses to propagate through the fibre with different central wavelengths. The GVD is obtained by dividing the group velocity differences by the wavelength shift. The nonlinear parameter $\gamma$ is determined using $\gamma=2 \pi n_{2} / \lambda A_{\text {eff }}$, where the nonlinearindex coefficient $n_{2} \sim 2.5 \times 10^{-20} \mathrm{~m}^{2} \mathrm{~W}^{-1}$ is used for silica. The two fibres are considered to be comparable since they have similar values for $\gamma L_{\text {eff. }}$.

As one of the most widely used low-cost standard single-mode fibre, LEAF fibre offers great compatibility and would substantially lower the cost of SBS slow light devices, rendering it the first candidate for our experiment. It turns out, however, the zero-dispersion wavelength for the LEAF is not shifted to the transparency window, resulting in a large anomalous dispersion $\beta_{2}$ at $\sim 1.55 \mu \mathrm{m}$, which degrades the SBS slow light performance, as shown below.

The SBS gain as a function of $P_{\mathrm{i}}$ is measured for the LEAF fibre and is shown in figure 2(a). As $P_{\mathrm{i}}$ is increased from zero to $0.8 \mathrm{~W}, G$ first scales linearly with $P_{\mathrm{i}}$, with a slope of $10.5 \mathrm{~W}^{-1}$, and then starts to saturate and deviate from linear growth at $G \sim 4$, corresponding to an input power $P_{\mathrm{i}} \sim 0.4 \mathrm{~W}$ (figure 2(a)). The early saturation of the SBS gain limits the SBS slow light delay.

The saturation behaviour shown in figure 2(a) could be the result of pump depletion due to the SBS process, as we mentioned previously. To rule out this effect, we note the following. First, the saturated value of the gain is $\sim 4$, which is much smaller than the threshold gain $G_{\text {th }}=$ 10 , indicating that saturation is not induced by spontaneous Brillouin amplification. Second, the amplified signal power $P_{\mathrm{S}}$ is only $0.5 \mathrm{~mW}$, which is small in comparison to the input pump power $0.75 \mathrm{~W}$, and is hence much too small to deplete the pump. Moreover, the total power $P_{\mathrm{o}}$ transmitted through the fibre in the direction of the pump beam grows linearly as $P_{\mathrm{i}}$ is increased from 0 to $0.8 \mathrm{~W}$, as shown in figure 2(b), which shows directly that the total transmitted power is not depleted.

To explain the early SBS gain saturation at $G \sim 4$, we examine the transmitted pump spectrum $p_{\mathrm{o}}$ passing through the $20 \mathrm{~km}$-long LEAF fibre (Anritsu model MS9710B optical spectrum analyzer). As $P_{\mathrm{i}}$ is increased from zero to $0.8 \mathrm{~W}$, no significant Raman gain is observed (figure 3(a)). On the other hand, in spectral span of $10 \mathrm{~nm}$, symmetric side lobe structures 

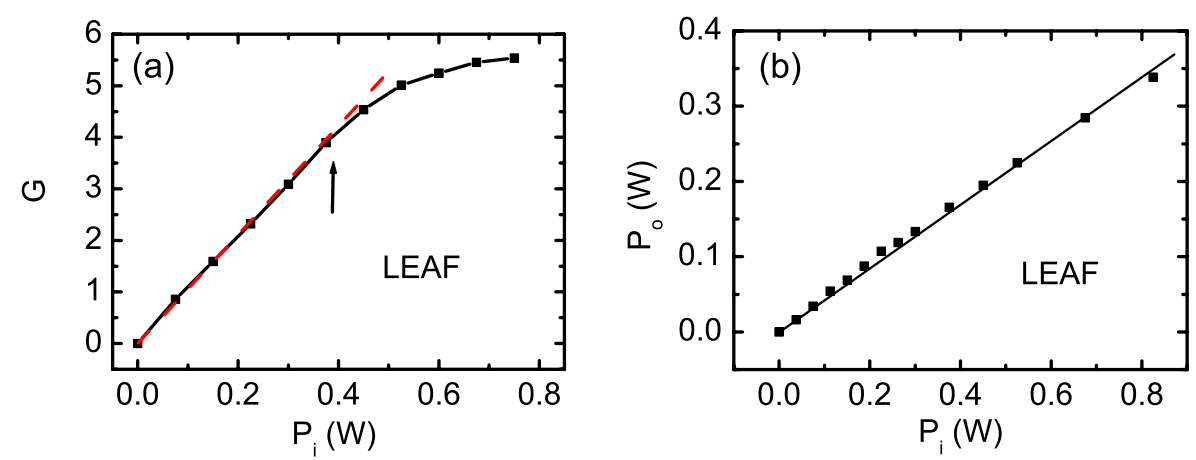

Figure 2. Early saturation of the SBS gain. (a) The SBS gain $G$ as a function of input pump power $P_{\mathrm{i}}$. Saturation is observed around $G \approx 4$ (vertical arrow), corresponding to an input pump power of $\sim 0.4 \mathrm{~W}$; (b) total power $P_{\mathrm{o}}$ transmitted through the fibre in the pump-beam direction (not spectrally resolved) as a function of the input pump power $P_{\mathrm{i}}$, indicating high fibre transparency. In the experiment, a weak continuous-wave beam with an input power of $2 \mu \mathrm{W}$ is used as the signal beam.
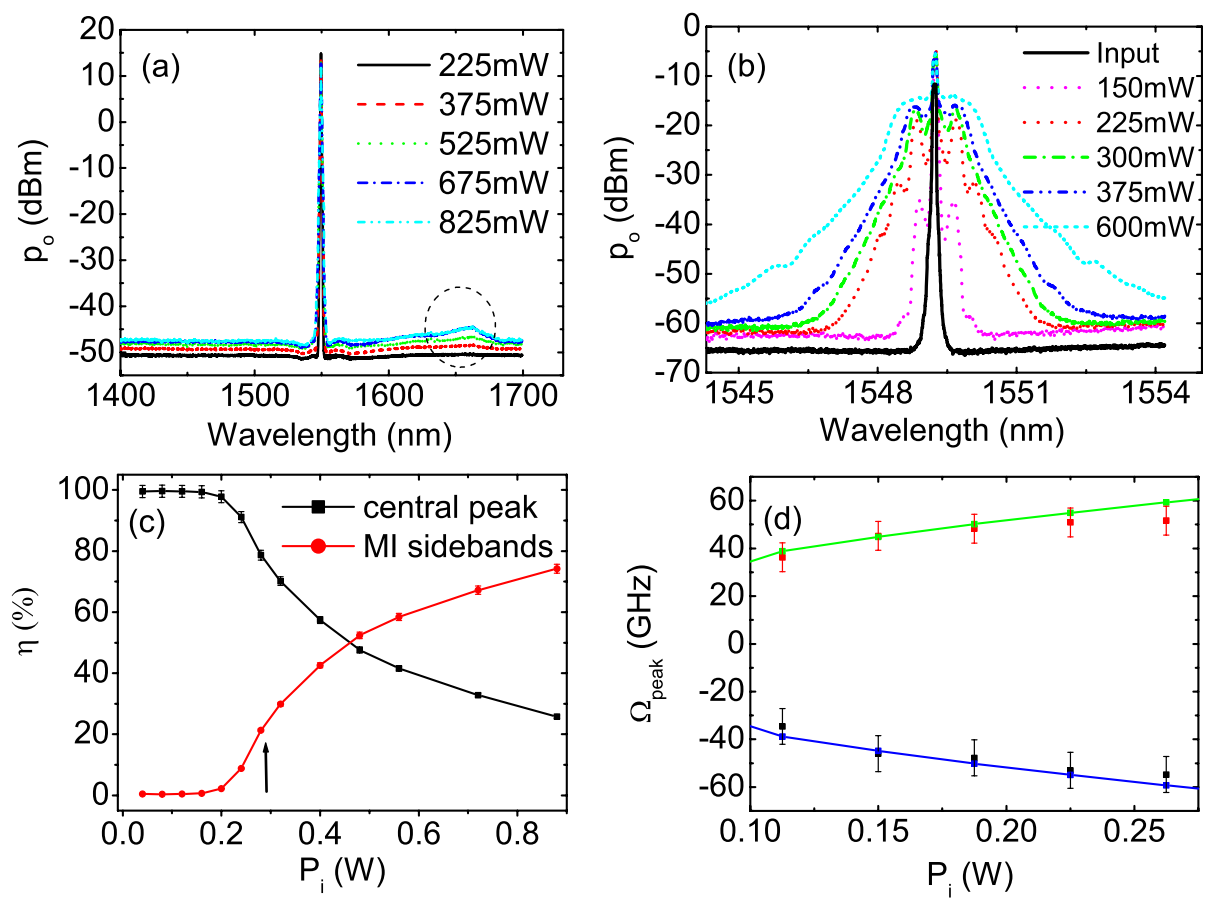

Figure 3. Modulation instability in the LEAF fibre. (a) Output power spectral density $p_{\mathrm{o}}$ of the pump laser (span of $300 \mathrm{~nm}$ ) with increasing input power $P_{\mathrm{i}}$. The circle indicates the Raman peak. (b) Output power density spectra $p_{\mathrm{o}}$ of the pump laser (span of $10 \mathrm{~nm}$ ) with increased input power $P_{\mathrm{i}}$. The input spectrum of the pump laser (at $40 \mathrm{~mW}$ ) is also shown. The resolution of the spectrum analyser is $0.1 \mathrm{~nm}$.

(c) Percentage $(\eta)$ of power distributed in the central peak (black square) and in the MI sidebands (red circle) for the output pump beam, as functions of the input power $P_{\mathrm{i}}$. The arrow indicates threshold at $\sim 0.3 \mathrm{~W}$. (d) Experimental data (point) and theoretical prediction (line) of the frequency shift $\Omega_{\text {peak }}$ of the MI side lobe peaks as a function of input power $P_{\mathrm{i}}$.

emerge and grow quickly as $P_{\mathrm{i}}$ increases (figure 3(b)). At high $P_{\mathrm{i}}$, the spectrum of the pump is flattened as a result of the emergence of secondary sidebands, and the power is spread into a broad frequency span of $\sim 200 \mathrm{GHz}$. Notice that the pump power transferred to the side lobes is no longer on resonance with the signal beam in the SBS interaction and therefore does not contribute to the SBS gain process.

To determine the amount of power that is transferred to the side lobes, we integrate the power spectral density $p_{\mathrm{o}}$ of the output pump beam and calculate the distracted power as percentages $\eta$ of the total power. As shown in figure $3(\mathrm{c})$, a considerable proportion $(>20 \%)$ of the pump power is transferred to the sidebands when the input power exceeds $P_{\mathrm{i}} \sim 0.3 \mathrm{~W}$. We define this point as the threshold for the MI process and the corresponding input pump power as the threshold power $P_{\mathrm{th}}^{\mathrm{MI}}$ in the LEAF fibre. The threshold gain $G_{\text {th }}^{\mathrm{MI}}$ is obtained by $(15)$ to be $\sim 10$. Note that $P_{\mathrm{th}}^{\mathrm{MI}}$ is close to the location where the early saturation of SBS gain occurs. We also compare the measured frequency shifts $\Omega_{\text {peak }}$ of the MI sidelobe peaks with the theoretical prediction using equation (16) and obtain good agreement, as shown in figure 3(d). These observations lead us to conclude that the strong saturation of the SBS gain is caused by the MI-induced pump broadening. In conclusion, we find that MI dominates 

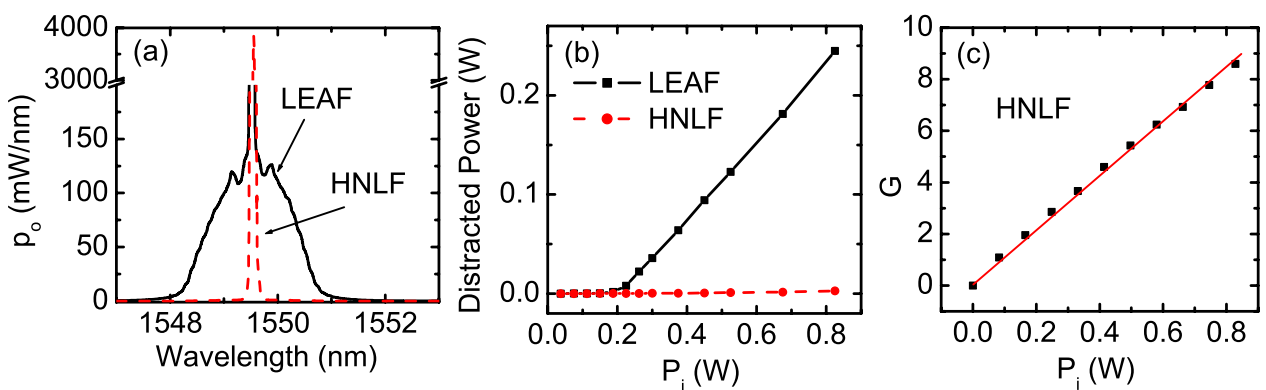

Figure 4. Suppression of modulation instability in HNLF. (a) Output power spectrum $p_{\mathrm{o}}$ of HNLF (red dash) and LEAF (black solid) at an input power of $0.8 \mathrm{~W}$; (b) MI distracted pump power as a function of input power $P_{\mathrm{i}}$ in HNLF (red dot) and LEAF (black square).(c) SBS gain $G$ in HNLF as a function of the input power $P_{\mathrm{i}}$.
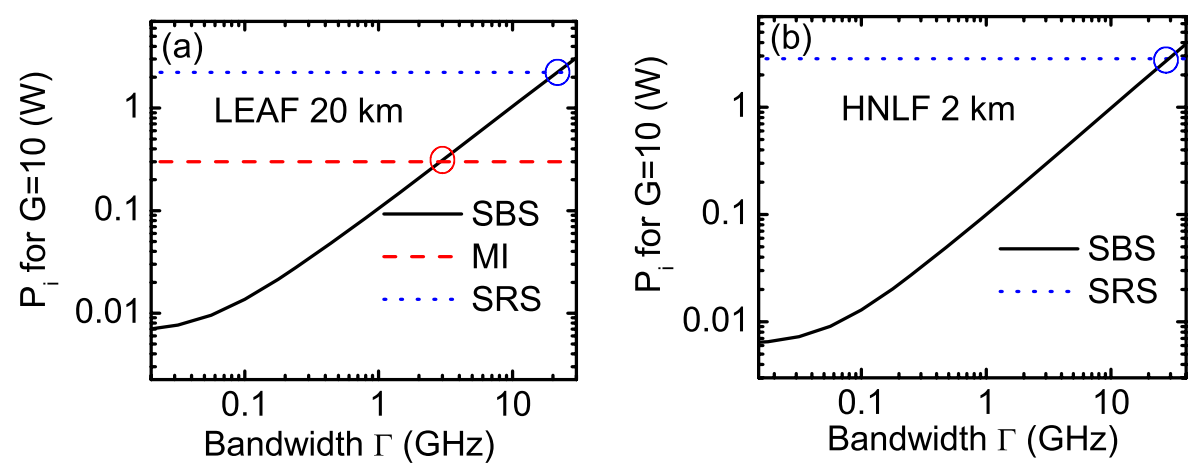

Figure 5. Pump power $P_{\text {i }}$ required for a $G$ of 10 versus the bandwidth $\Gamma$.

over SBS beyond a threshold pump power of $P_{\mathrm{th}}^{\mathrm{MI}}=0.3 \mathrm{~W}$ in the LEAF fibre. In a broadband system where $\Gamma=9.2 \mathrm{GHz}$, the threshold pump power corresponds to an SBS gain $G \sim$ 3.2, which leads to a limit of $29 \mathrm{GHz}$ or $125 \mathrm{~dB} \mathrm{GHz}$ on the SBS gain-bandwidth product.

We next turn to the dispersion-shifted HNLF fibre, which has a small and normal dispersion at $1.55 \mu \mathrm{m}$. Because it is widely known that the MI is suppressed due to phase matching constraints in normally dispersive materials [14, 20, 23], we expect to see suppression of the MI and improvement in the gain-bandwidth product for the broadband SBS slow light with the HNLF fibre. As shown in figure 4, it is found that the MI-induced limit on the SBS gain-bandwidth product is indeed removed. With the same $9.2 \mathrm{GHz}$ broadband pump input, the transmitted pump spectrum through the HNLF shows no significant MI peaks (figure 4(a)). The power converted into off-resonant frequencies is negligible (figure 4(b)). As expected, early saturation of the SBS gain in the HNLF does not appear (figure 4(c)), resulting in a larger SBS gainbandwidth product of $344 \mathrm{~dB} \mathrm{GHz}$, limited by our available pump power of $0.82 \mathrm{~W}$. The result further confirms that MIinduced pump broadening is the origin of the early saturation of SBS gain in the LEAF fibre.

\section{Discussion}

In this section, we compare the relative importance of the two effects that compete with SBS in our broadband SBS slow light system. Following the approach of Olsson et al, we compare the pump power $P_{\mathrm{i}}$ requirements for the broadband SBS, SRS, and MI processes in the LEAF fibre. Figure 5 shows the input pump power $P_{\mathrm{i}}$ required to obtain a threshold gain of 10 as a function of the bandwidth $\Gamma$. The threshold pump power for the SBS process is obtained from equation (12) taking $G=10$ and $g_{0}=4.35 \times 10^{-12} \mathrm{~m} \mathrm{~W}^{-1}$ (obtained from figure 2(a)), giving

$$
P_{\mathrm{i}}=G \frac{\Gamma}{2 \Gamma_{\mathrm{B}}} \frac{A_{\mathrm{eff}}}{g_{0} L_{\mathrm{eff}}} \arctan \left(\Gamma / \Gamma_{\mathrm{B}}\right)^{-1} .
$$

Note that we assume that a weak probe beam is used so that the probe-induced SBS saturation does not appear.

The SRS gain is given by [14]

$$
G_{\mathrm{SRS}}=2 g_{R} P_{\mathrm{i}} L_{\mathrm{eff}} / A_{\text {eff }},
$$

where $g_{R}=1.26 \times 10^{-14} \mathrm{~m} \mathrm{~W}^{-1}$ [15]. The threshold power required for an SRS gain of 10 is obtained by $P_{\mathrm{i}}=$ $10 A_{\text {eff }} /\left(2 g_{R} L_{\text {eff }}\right)=2.23 \mathrm{~W}$. The threshold pump power of the MI process is found to be $\sim 0.3 \mathrm{~W}$, as mentioned previously.

As shown in figure 5(a), beyond the bandwidth of $\sim 2.9 \mathrm{GHz}$, the LEAF-fibre system hits the MI threshold before it saturates the SBS gain. As a result, MI sets a limit to the SBS gain-bandwidth product. The gain-bandwidth product limit is $29 \mathrm{GHz}$, or $125 \mathrm{~dB} \mathrm{GHz}$ using $G=10$ in the LEAF fibre. Figure 5 also shows that the SRS becomes more efficient than the SBS when the bandwidth goes beyond $\sim 22 \mathrm{GHz}$, and limits the SBS gain-bandwidth product up to $217 \mathrm{GHz}$ or $941 \mathrm{~dB}$ GHz. The result is consistent with what Olsson et al predict [15]. In the LEAF fibre, the SBS gain-bandwidth product is restricted by the tighter MI-induced limit. 
A similar plot is made for the HNLF fibre, as shown in figure 5(b). We see that in this normally dispersive fibre where the MI is suppressed, SRS becomes the main restriction. The threshold power required for an SRS gain of 10 in the HNLF fibre is obtained to be $2.83 \mathrm{~W}$, which results in the suppression the SBS process beyond the bandwidth of $\sim 29 \mathrm{GHz}$ and limits the SBS gain-bandwidth product up to $290 \mathrm{GHz}$, or $1.26 \mathrm{~dB} \mathrm{THz}$, consistent with our measured SBS gain-bandwidth product of $344 \mathrm{~dB} \mathrm{GHz}$ in this fibre.

In conclusion, we found that in the broadband SBS systems, MI dominates in the high pump power region and sets a limit to the SBS gain-bandwidth product, which is 125 $\mathrm{dB} \mathrm{GHz}$ in the LEAF fibre. The SBS suppression and SBS slow light performance is improved in normally dispersive materials.

\section{Acknowledgment}

We gratefully acknowledges the financial support of the DARPA Defense Sciences Office Slow Light project.

\section{References}

[1] Boyd R and Gauthier D 2009 Science 3261074

[2] Gonzalez-Herraez M, Song K and Thevenaz L 2005 Appl. Phys. Lett. 87081113

[3] Okawachi Y, Bigelow M, Sharping J, Zhu Z, Schweinsberg A, Gauthier D, Boyd R and Gaeta A 2005 Phys. Rev. Lett. 94153902

[4] Tucker R, Ku P and Chang-Hasnain C 2005 J. Lightwave Technol. 234046
[5] Bo Z, Yan L, Yang J, Fazal I and Willner A 2007 IEEE Photon. Technol. Lett. 19 1081-3

[6] Cabrera-Granado E, Calderon O, Melle S and Gauthier D 2008 Opt. Express 16 16032-42

[7] Stenner M, Neifeld M, Zhu Z, Dawes A and Gauthier D 2005 Opt. Express 13 9995-10002

[8] Shi Z, Pant R, Zhu Z, Stenner M, Neifeld M, Gauthier D and Boyd R 2007 Opt. Lett. 32 1986-8

[9] González Herráez M, Song K and Thévenaz L 2006 Opt. Express 14 1395-400

[10] Song K and Hotate K 2007 Opt. Lett. 32 217-9

[11] Yi L, Jaouen Y, Hu W, Su Y and Bigo S 2007 Opt. Express 15 16972-9

[12] Zhu Z, Dawes A, Gauthier D, Zhang L and Willner A 2007 J. Lightwave Technol. 25 201-6

[13] Stolen R, Gordon J, Tomlinson W and Haus H 1989 J. Opt. Soc. Am. B 6 1159-66

[14] Agrawal G 2001 Nonlinear Fiber Optics (Berlin: Springer)

[15] Olsson N and Van Der Ziel J 1987 J. Lightwave Technol. 5 147-53

[16] Boyd R 2008 Nonlinear Optics (Amsterdam: Elsevier)

[17] Boyd R, Gauthier D, Gaeta A and Willner A 2005 Phys. Rev. A 7123801

[18] Zhu Z, Gauthier D, Okawachi Y, Sharping J, Gaeta A, Boyd R and Willner A 2005 J. Opt. Soc. Am. B 22 2378-84

[19] Boyd R, Rzaewski K and Narum P 1965 Phys. Rev. A 425514

[20] Dianov E, Mamyshev P, Prokhorov A and Chernikov S 1989 Opt. Lett. 14 1008-10

[21] Cavalcanti S, Agrawal G and Yu M 1995 Phys. Rev. A 51 4086-92

[22] Mussot A, Lantz E, Maillotte H, Sylvestre T, Finot C and Pitois S 2004 Opt. Express 12 2838-43

[23] Tai K, Hasegawa A and Tomita A 1986 Phys. Rev. Lett. 56 135-8

[24] Cohen L and Lin C 1977 Appl. Opt. 16 3136-9 